\title{
EDITORIAL
}

\section{Advancing organizational behavior through context considerations}

It gives me a great pleasure to introduce this issue of Journal of Management \& Organization, which brings together a collection of organizational behavior articles incorporating context as a variable. The first article in the issue, following this editorial, provides a review of the consideration of context in organizational behavior research, highlighting the under-appreciation and under-representation of the important role that context has in the development of innovative and useful organizational behavior theory and practice. The article, entitled 'Contextualizing research: Putting context back into organizational behavior research,' provides examples and guidance for taking advantage of the valuable insights that a consideration of context can provide to organizational life. It is my sincere hope that the collections of articles in this issue will spur you to consider how context can inform your research, teaching and practice in organizational behavior.

Following the article by Härtel and O'Connor, is our invited contribution. This special article by Blake Ashforth and colleague Glen Kreiner exemplifies how considerations of context can enhance our understanding and theorizing about organizational behavior. Their article, entitled 'Contextualizing dirty work: The neglected role of cultural, historical, and demographic context' gives us new insight into occupation as context. Our eyes are opened to seeing that some occupation contexts are characterized by stigma. As Blake and Glen point out, there is a need to understand how it is that some types of work come to be seen as 'dirty' and how workers in such occupations make sense of their work. Not only is occupation seen as context, but so is time, with a historical analysis providing insight on how shifts in societal context features such as hygiene, economics, technology, culture, and politics, correspond with shifts in the stigma attached to certain occupations.

Next in line is the article entitled 'Personality and contextual antecedents of organizational citizenship behavior: A study of two occupational groups.' This article, by Imer, Kabasakal and Dastmalchian, also examines occupation as context. They point out that occupation as a context has been shown in a number of studies to predict organizational citizenship behaviors. In their study, they identify unique features of the organizational citizenship behaviors exhibited by workers in the context of a teaching occupation versus an engineering occupation.

Moving on to relationships as context, the article entitled 'Subordinates' feedback-seeking behavior in supervisory relationships: A moderated mediation model of supervisor, subordinate, and dyadic characteristics,' identifies features of the leader-follower dyad context that influence the likelihood a subordinate will engage in feedback-seeking behavior. Interestingly, they show that when a specific personality configuration of follower self-monitoring and leader credibility is present, there is a shift in the perceived cost of feedback-seeking, which in turn, impacts a follower's decision to seek performance feedback.

Also looking at relationships as context, the article entitled 'The relationship between workgroup identification and organizational identification: The moderating role of perceived similarities between targets,' considers contexts where there are perceived similarities and where there are perceived dissimilarities between workgroups and the organization. This study identifies that the relationship between workgroup identification and organizational identification depends on whether the context is one of perceived similarities or perceived dissimilarities in the workgroup-organization relationship.

The next article entitled 'How does self-regulation of emotions impact employee work engagement: The mediating role of social resources,' considers social resources as context. In particular, the study 
provides evidence that self-regulation of emotions is not always enough to facilitate work engagement. The context of an individual's social resources can override the effects of self-regulation.

In the article that follows, 'The role of voice efficacy in the formation of voice behavior: A crosslevel examination,' perceived organizational support is considered as a contextual influence on voice behavior. The findings reveal that self-efficacy has a stronger relationship to voice behavior when organizational support is perceived as high.

The last article in this issue, entitled 'Delving into promises: Conceptually exploring the beliefs constituting the contemporary psychological contract,' looks at the psychological contract as context. In particular, by reviewing literature from multiple disciplines, novel ways of contextualizing the psychological contract are identified.

Collectively, the articles in this issue provide evidence of the value of considering context in organizational behavior research as well as identifying ways in which organizational behavior scholars can incorporate contextual considerations in their research designs.

Charmine E J Härtel Senior Editor Journal of Management \& Organization UQ Business School The University of Queensland Brisbane, QLD 4072 Australia 